\title{
Applications of High Frequency Eddy Current Technology for Material Characterization of Thin Coatings
}

\author{
Oliver Bruchwald*, Wojciech Frackowiak, Wilfried Reimche and Hans Jürgen Maier \\ Institut für Werkstoffkunde, Leibniz Universität Hannover, D-30823 Garbsen, Germany
}

\begin{abstract}
Thin metallic coatings are widely used for corrosion protection or to improve the functional and wear properties of the base material. Because these coatings can wear out and lose their functionality during lifetime, a non-destructive characterization of the coating condition is needed. The eddy current technology allows for a non-destructive characterization of metallic coatings as well as measuring the coating thickness of both metallic or non-metallic, non-conductive coatings. However, very thin coatings with a low electrical conductivity, e.g. the PtAl- or MCrAlY-coatings typically deposited on turbine blades, cannot be characterized separately from the metallic base material due to the high penetration depth when using conventional eddy current technology with testing frequencies below $5 \mathrm{MHz}$. Experimental investigations were carried out using a new high frequency eddy current testing (HF-EC) system with a frequency range between 5 and $100 \mathrm{MHz}$ and test samples with zinc coatings between 10 and $45 \mu \mathrm{m}$ as well as high pressure turbine blades made of nickel-base alloys with a PtAl coating and a non-conductive thermal barrier coating TBC. It will be shown that the HF-EC technology allows for a quick estimation of the thin coating's condition and its thickness by analyzing the local electric properties and the lift-off effect.
\end{abstract}

Key words: High frequency eddy current testing, material characterization, coating condition, thin coatings, turbine blades.

\section{Introduction}

Turbine blades in modern aircraft engines are exposed to high temperatures, mechanical loads and corrosion. Therefore, a metallic corrosion protective coating as well as a ceramic thermal barrier coating (TBC) is necessary to protect the base material of the first stage turbine blades.

During lifetime the turbine blades TBC thickness can be reduced by erosion or spalling. In addition, the local chemical composition of the underlying corrosion protective layer also changes, especially in highly loaded areas $[1,2]$. Unfortunately, the individual and non-destructive characterization of the base material, the aluminium-based corrosion protective coating condition and the TBC thickness

\footnotetext{
${ }^{*}$ Corresponding author: Oliver Bruchwald, Ph.D. student and scientific assistant, research fields: non-destructive characterization of thin coating systems and heat treatment processes.
}

are difficult using conventional eddy current technology due to the very thin coating thicknesses between 20 and $100 \mu \mathrm{m}$, and the significantly higher eddy current penetration depth.

The eddy current penetration depth depends mainly on the magnetic permeability, the electric conductivity and the test frequency. Therefore thin metallic coatings with a given low magnetic permeability and low electric conductivity can only be characterized separately from the metallic base material by using high test frequencies [3, 4], see Fig. 1.

Within the framework of the collaborative research centre CRC 871 a new high frequency eddy current (HF-EC) test system with a frequency range of up to $100 \mathrm{MHz}$ was developed to characterize thin coatings, especially with a focus on the multilayer coating systems of turbine blades [5]. This paper shows some representative results using eddy current testing methods to characterize different coating combinations 


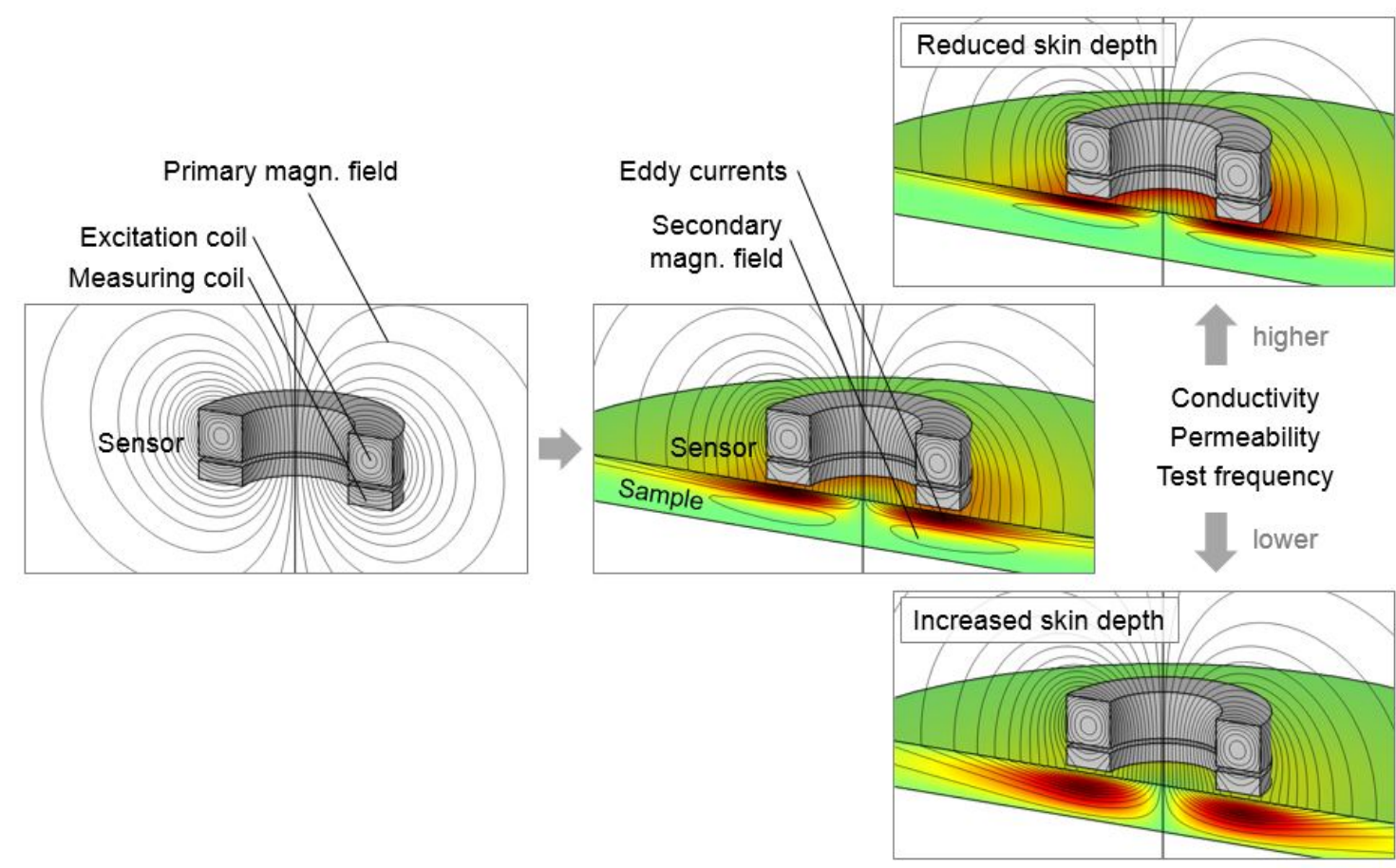

Fig. 1 Influence of the conductivity, the permeability and the test frequency on the eddy current penetration depth.

as well as high pressure turbine blades with a PtAl coating and a non-conductive TBC.

\section{Coating Characterization Using Eddy Current Technology}

The eddy current technology can be used to characterize three basic coating configurations:

- Non-metallic, non-conductive coatings on a metallic base material.

- Metallic coatings on a non-metallic, non-conductive base material.

- Metallic coatings on a metallic base material.

\subsection{Coating Thickness of Non-Metallic Coatings on a Metallic Base Material}

The material characterisation using eddy current technology is based on the induction of eddy currents in the electroconductive part of the sample and the resulting secondary magnetic field, which generates a characteristic signal in the measuring coil. Therefore non-metallic, non-conductive coatings cannot be directly characterized. However, eddy currents are induced in the electroconductive base material and the coating leads to a non-conductive gap between the sensor and the base material. With an increasing distance between the base material and the sensor the primary magnetic field and the eddy currents in the base material become weaker, and thus, the voltage induced in the measuring coil is reduced (lift-off-effect).

Fig. 2 shows the influence of the non-conductive coating thickness on the measured signal in the impedance plane. The highest amplitude is achieved while the sensor is in direct contact with the metallic base material. Depending on the coating thickness the amplitude decreases and would reach $0 \mathrm{~V}$ for an infinite coating thickness and a sensor that was compensated in air. As seen in Fig. 2, the signal is very sensitive to distance, and thus, the lift-off-effect can be exploited to accurately determine the coating thickness of non-metallic coatings on a metallic base material [6].

2.2 Coating Thickness and Coating Condition of Metallic Coatings on a Non-Conductive Base Material

When testing a very thin metallic coating on a 


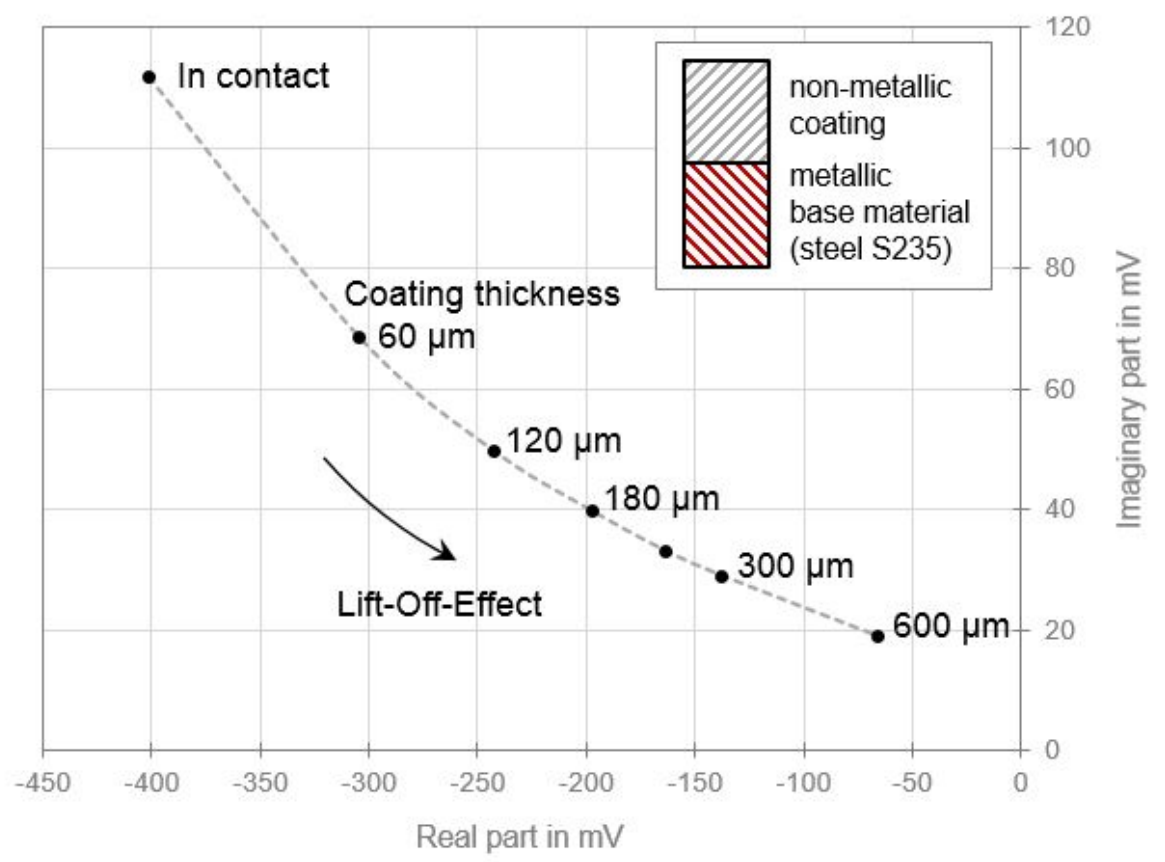

Fig. 2 Determination of the non-metallic coating thickness by analyzing the lift-off-effect.

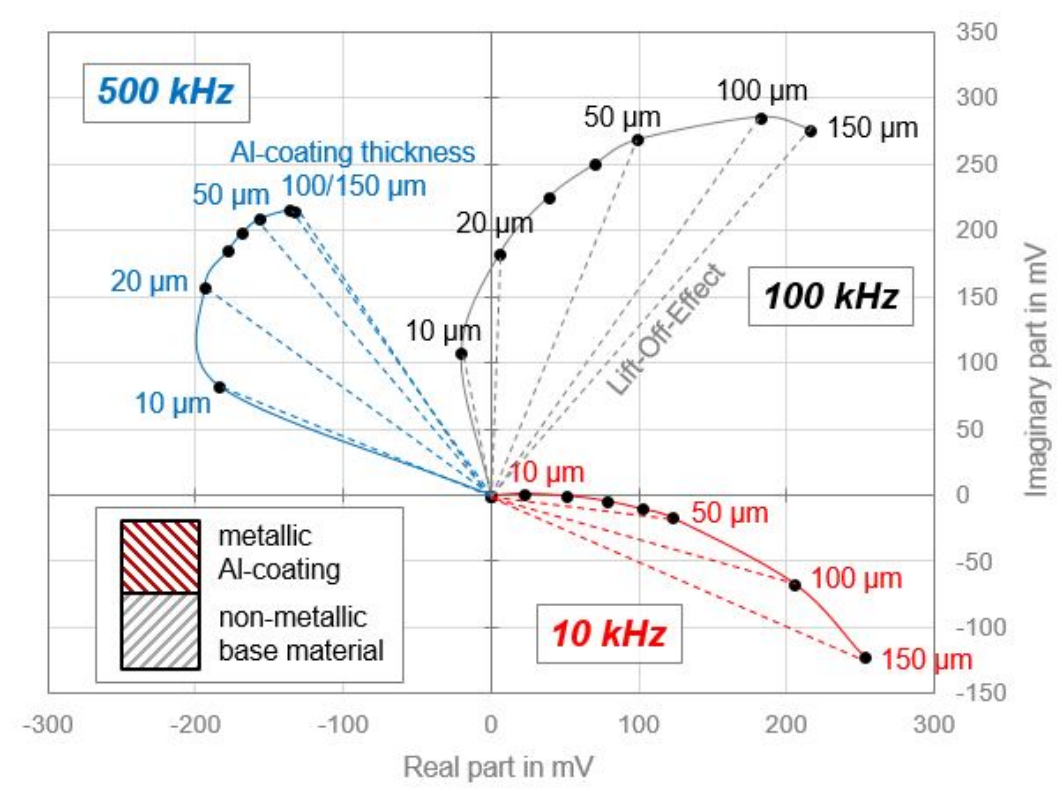

$$
\delta=\sqrt{\frac{1}{2 \cdot \pi \cdot f \cdot \mu \cdot \sigma}}
$$

Aluminium coating

Conductivity:

$\sigma=37.5 \mathrm{MS} / \mathrm{m}$

Permeability:

$\mu_{\mathrm{r}}=1$

Test frequency:

$\mathrm{f}=10,100,500 \mathrm{kHz}$

Skin depth:

$\delta=800,250,120 \mu \mathrm{m}$

Fig. 3 Determination of the metallic coating thickness on a non-metallic substrate for three different test frequencies.

non-metallic, non-conductive base material with low test frequencies, the formation of eddy currents in the coating is usually restricted by its thickness. Therefore an increasing coating thickness leads to stronger eddy currents in the coating. This results in a characteristic change in the measured signal and a higher signal amplitude.
Fig. 3 shows data obtained for thin aluminium coatings on a non-metallic base material with three different test frequencies. The skin depth or standard penetration depth $\delta$ of the eddy currents can be estimated using the equation shown in the inset [2].

Using a test frequency of $10 \mathrm{kHz}$, coating thicknesses in the range between 10 and $150 \mu \mathrm{m}$ can 
be reliably determined from the signal due to the high penetration depth of approx. $800 \mu \mathrm{m}$, which by far exceeds the coating thickness. However, due to the great influence of the coating thickness on the measured signal, a reliable characterization of the coating condition based on slight changes in the electrical conductivity is extremely difficult in this case.

At a test frequency of $500 \mathrm{kHz}$ the penetration depth is reduced to approx. $120 \mu \mathrm{m}$. Coatings with a thickness greater than the penetration depth do not influence the measured signal any further due to the undisturbed eddy current formation. Therefore, the signal recorded from the $100 \mu \mathrm{m}$ and the $150 \mu \mathrm{m}$ Al-coating (with identical properties) is also nearly identical when slowly approaching the sensor towards the sample (dashed line).

In summary, the coating thickness of metallic coatings on a non-metallic base material can be estimated by using low test frequencies due to characteristic changes in the measured signal when the penetration depth is greater than the coating thickness. For a characterization of the coating condition, however, higher frequencies resulting in a penetration depth lower than the coating thickness should be used to suppress the influence of varying coating thicknesses.

\subsection{Coating Thickness and Coating Condition of Metallic Coatings on a Metallic Base Material}

When testing metallic coatings on a metallic base material, eddy currents are induced in both materials and the resulting signal consists of two superimposed contributions - one from the base material and one from the coating. Depending on the test frequency and the coating thickness, the individual contributions to the measured signal will change. Therefore coating thicknesses can only be estimated if the penetration depth is greater than the coating thickness and if both materials feature sufficiently different magnetic or electrical properties.

Fig. 4 shows the measured signal for an aluminium coated structural steel sample. An increasing coating thickness (black curve) leads to a characteristic change in the signal due to the higher concentration of eddy currents in the coating and a lower concentration in the base material. When the coating thickness reaches $150 \mu \mathrm{m}$ the base material has only a negligible effect on the measured signal because the eddy currents are mainly concentrated in the aluminium coating. Therefore, the signal is almost identical with the one obtained from the Al-coating on the non-metallic base material (red dot), see Fig. 3 .

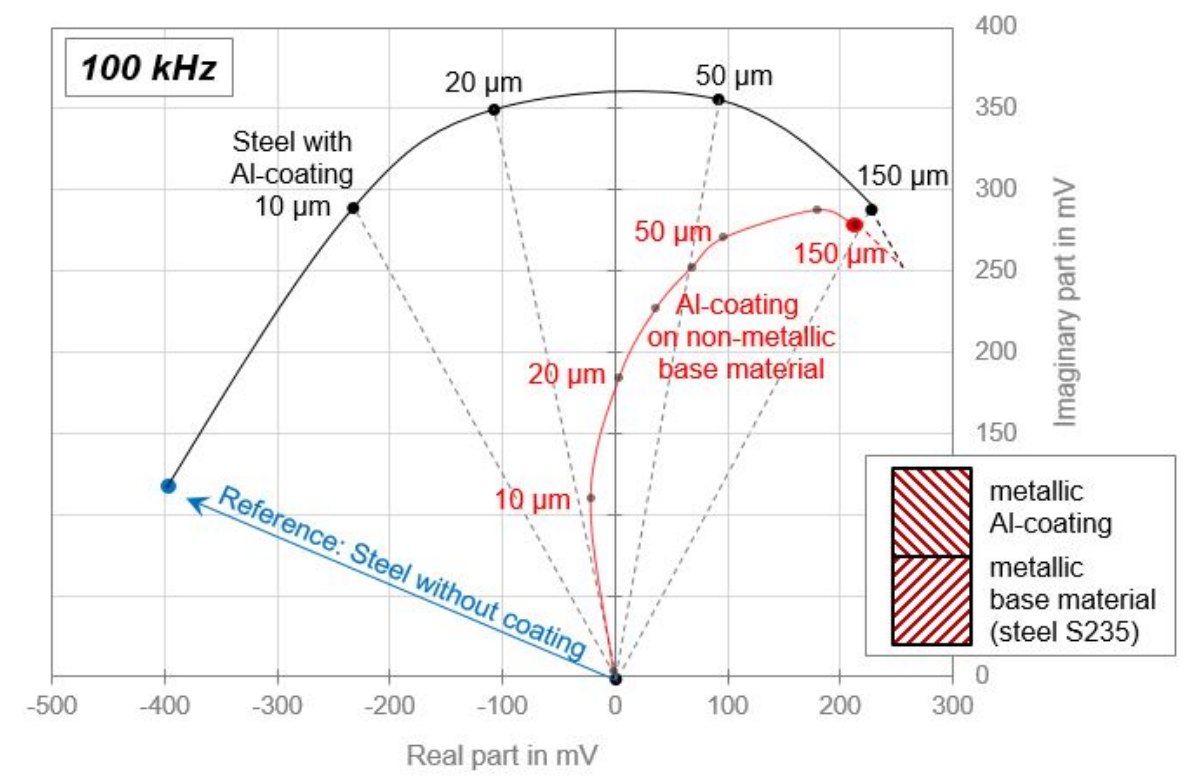

Fig. 4 Determination of the metallic coating thickness on a metallic base material. 
Fig. 5 shows an anti-corrosion zinc-coating with a thickness between 10 and $45 \mu \mathrm{m}$ on a structural steel sample and the corresponding measured signal. Due to the electrical conductivity of zinc with approx. 16 $\mathrm{MS} / \mathrm{m}$ the penetration depth is higher than the coating thickness when using conventional eddy current testing systems with a maximum test frequency of 5 MHz. Therefore, a reliable characterization of the coating condition (e.g. the coating density, porosity, quality) that correlates with slight changes in the electrical conductivity is only possible using high frequency eddy current testing. As can be seen, a test frequency of $25 \mathrm{MHz}$ generates eddy currents with a max. depth of approx. $25 \mu \mathrm{m}$, so that both samples with thicker coatings ( $28 \mu \mathrm{m}$ and $45 \mu \mathrm{m})$ cause almost identical measured signals and the influence of the coating thickness and the metallic base material vanishes.

In summary, metallic coatings on a metallic base material lead to a more complex signal that can have contributions from both the coating and the substrate. The coating thickness can be estimated if both materials feature sufficiently different magnetic or electrical properties and the penetration depth is greater than the coating thickness. With higher testing frequencies the influence of the coating thickness and the base material on the measured signal can be suppressed.

\section{Characterization of the Turbine Blades Coating System}

The investigated first stage high pressure turbine blades were made of a nickel-base alloy and featured a multilayer coating system with a PtAl-coating and a ceramic thermal barrier coating. It is well known that during its lifetime the corrosion protective layer's chemical composition changes in the highly loaded areas $[1,2]$. The metallic components of the coating convert into oxides, and thus, cause a reduction in the electrical conductivity that can be used to estimate the coating condition. Due to the very low electrical conductivity of the PtAl-coatings ( $\sim 1.5 \mathrm{MS} / \mathrm{m})$ and a typical layer thickness between 20 and $100 \mu \mathrm{m}$ the test frequency has to exceed $20 \mathrm{MHz}$ if the coating should be characterized separately from the base material and if the influence of the coating thickness should be supressed [5]. Furthermore the TBCs thickness deposited on top of the PtAl can be reduced by erosion or spalling. This in turn leads to a change in the non-conductive gap between the sensor and the

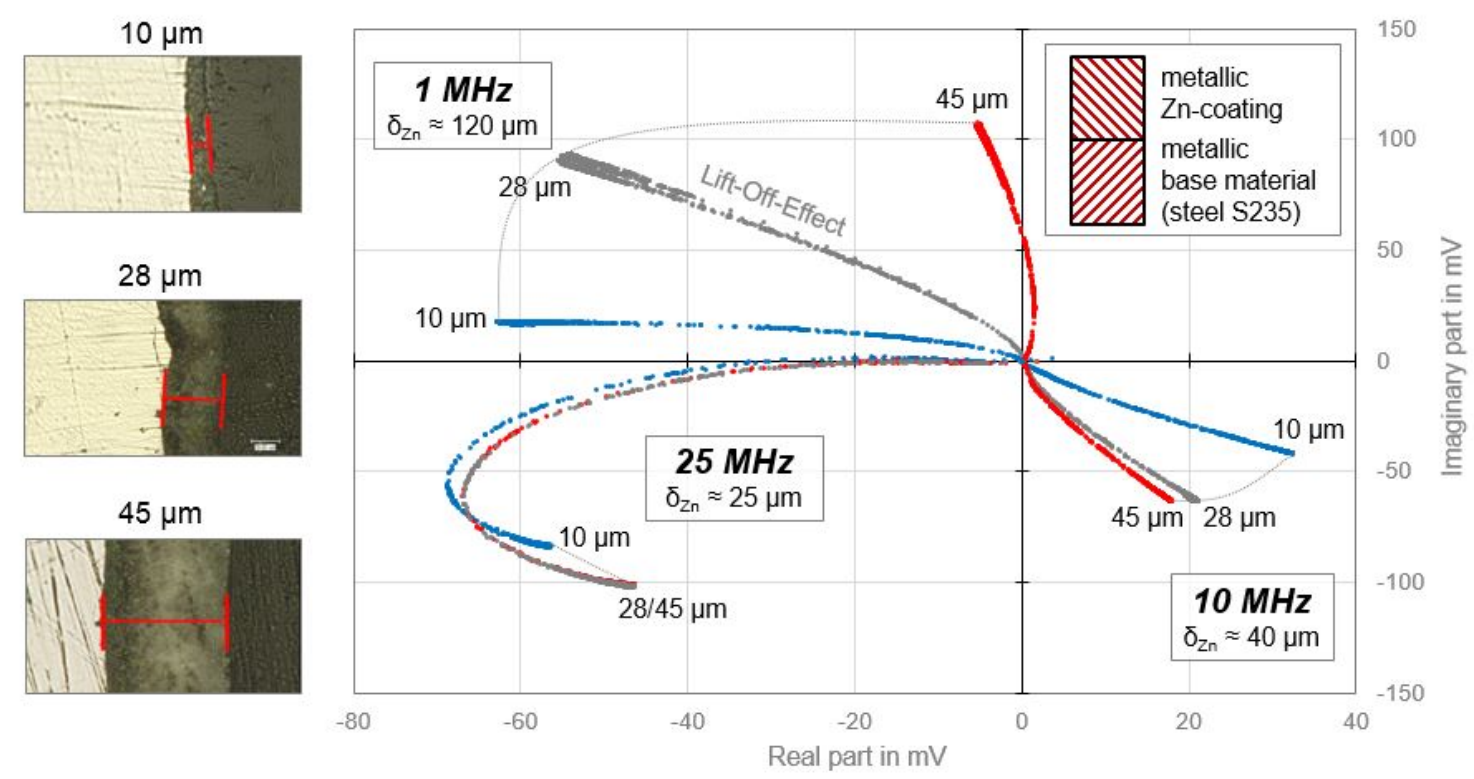

Fig. 5 Determination of the thickness of a zinc anti-corrosion coating on a steel substrate. 


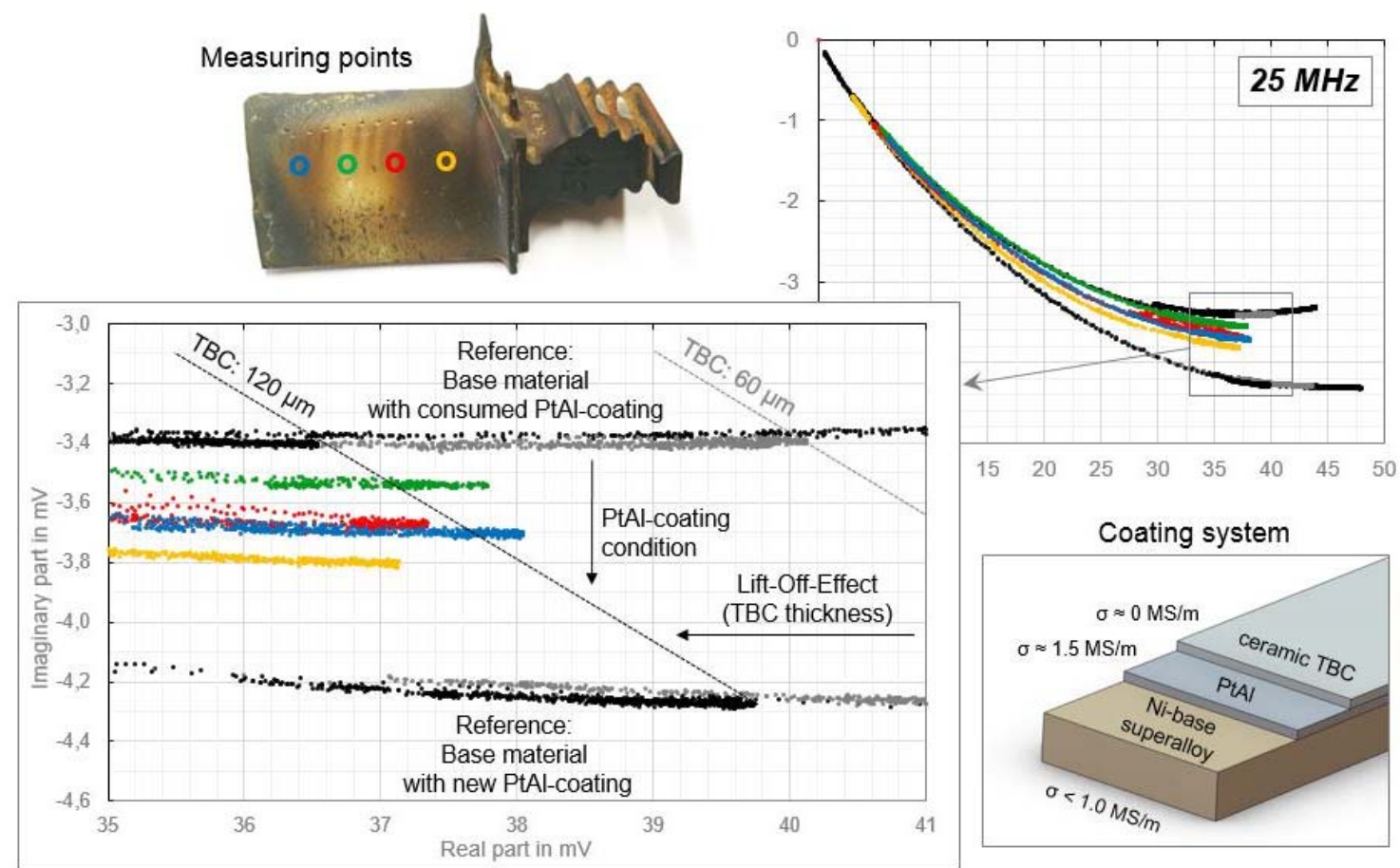

Fig. 6 Characterization of coating systems on turbine blades exposed to different local loading scenarios; see main text for details.

PtAl-coating and can be measured by analysing the lift-off-effect [6].

Fig. 6 depicts a turbine blade that exhibits regions of different loading conditions over the blade's height. The measured signals during slowly approaching the sensor towards the four measuring points (yellow, red, green and blue) on the turbine blades surface are shown in the impedance plane. Furthermore the behaviour of two blades with a new and a consumed PtAl-coating and different TBC coating thicknesses of 0,60 and $120 \mu \mathrm{m}$ are shown as a reference (black/grey).

The lift-off-effect caused by the TBC thickness, leads to changes in the real part whereas the PtAl-coating condition, characterised by the electric conductivity, leads to changes in the imaginary part of the measuring signal. In this example, a TBC thickness of $\sim 130 \mu \mathrm{m}$ in the turbine blades foot region (yellow, low service loads) and $\sim 110 \mu \mathrm{m}$ in highly loaded area (green) could be measured. The condition of the underlying PtAl-coating can be classified as "consumed", especially in the highly loaded area.

\section{Conclusions}

Conventional eddy current technology and high-frequency eddy current technology was used to estimate the coating thickness of metallic and non-metallic coatings on metallic and non-metallic base materials as well as for characterization of the coating condition of thin metallic coatings, especially the multilayer coatings applied on high-pressure turbine blades. It was shown that also very thin coatings can be characterised separately from the base material using high test frequencies. Specifically it could be shown that:

The coating thickness of non-metallic coatings on a metallic base material can be estimated by the lift-off-effect.

The coating thickness of metallic coatings on a non-metallic or a metallic base material can be estimated when the penetration depth is higher than the coating thickness (low test frequencies).

The coating condition of metallic coatings on a non-metallic or a metallic base material can be reliably estimated when the penetration depth is 
lower than the coating thickness (high test frequencies).

A combined non-destructive characterization of a thin multilayer coating system (non-metallic coating thickness and metallic coating condition) is also possible with only one measurement.

\section{Acknowledgments}

The present study was conducted within the framework of the collaborative research centre 871 "Regeneration of complex capital equipment" in sub-project A1 "Non-destructive characterisation of coatings and material conditions of highly loaded aircraft engine components". Financial support of this project by the German Research Foundation (DFG) is gratefully acknowledged.

This paper was originally published in the proceedings of the THE "A" Coatings Conference [7].

\section{References}

[1] Bräunling, W. J. G. 2009. Flugtriebwerke. Springer-Verlag.

[2] Cosack, T. 2009. Schutzschichten auf Turbinenschaufeln im Flugtriebwerk. MTU AeroEngines Publikation.

[3] Hagemaier, D. 2013. "Eddy Current Depth of Penetration." Materials evaluation 62 (10): 1028-9.

[4] Campbell, F. C. 2013. Inspection of Metals. ASM International.

[5] Reimche, W., Bruchwald, O., Frackowiak, W., Bach, Fr. -W. and Maier, H. J. 2012. "Non-Destructive Determination of Local Damage and Material Condition in High-Performance Components." HTM J. Heat Treatm. Mat. 68 (2): 59-67.

[6] Scheer, C., Reimche, W., Möhwald, K. and Bach, Fr.-W. 2008. "Entwicklung einer Online Schichtdickenmessung für das Plasmaspritzen von Keramik auf Basis einer Wirbelstromsensorik." Schweissen und Schneiden 60 (6): 331-6.

[7] Bruchwald, O., Frackowiak, W., Reimche, W. and Maier, H. J. 2016. "Applications of High Frequency Eddy Current Technology for Material Characterization of Thin Coatings." In Proceedings of the THE "A" Coatings Conference 2016, 37-43. 